\title{
Detecting and Visualizing the Communities of Innovation in Beijing-Tianjin-Hebei Urban Agglomeration Based on the Patent Cooperation Network
}

\author{
Fang Zhou $\mathbb{D}^{1,2}$ and Bo Zhang ${ }^{3}$ \\ ${ }^{1}$ College of Urban Economics and Public Administration, Capital University of Economics and Business, Beijing 100070, China \\ ${ }^{2}$ Beijing Key Laboratory of Megaregions Sustainable Development Modeling, Beijing 100070, China \\ ${ }^{3}$ School of Environment \& Natural Resources, Renmin University of China, Beijing 100872, China \\ Correspondence should be addressed to Fang Zhou; zhoufang@cueb.edu.cn
}

Received 4 August 2020; Revised 17 December 2020; Accepted 19 December 2020; Published 5 January 2021

Academic Editor: Yi Su

Copyright (c) 2021 Fang Zhou and Bo Zhang. This is an open access article distributed under the Creative Commons Attribution License, which permits unrestricted use, distribution, and reproduction in any medium, provided the original work is properly cited.

\begin{abstract}
For a deep understanding of Beijing-Tianjin-Hebei (BTH) collaborative innovation, we detected and visualized the communities of innovation in BTH Urban Agglomeration based on the patent cooperation network. China Patent Database was connected with Business Registration Database and the Tianyan Check to achieve the geographical information of organizational innovators. Spinglass algorithm was applied and ultimately 12 communities of innovation were detected. Based on the different structure characteristics, we further clustered the 12 communities into four typical structures that are hierarchical, single-center, polycentric, and flat structures. The hierarchical structure is usually large in scale and the cooperative intensity is relatively high. Single-center structure has a center with a high proportion of centrality and the cooperative intensity is relatively low. Polycentric structure has multiple centers with similar proportions of centrality. Flat structure is usually small in scale and has no obvious network center. In the patent cooperative network of BTH Urban Agglomeration, universities and state-owned enterprises occupied the centers and acted important roles to connect other organizations. Some communities of innovation showed significant industry characteristics, mainly involving six industry fields that are electric power, construction, petroleum, metallurgy and materials, municipal transportation, and railway. From the geographical perspective, some communities manifested local attributes and some demonstrated cooperation between regions. Beijing was the center of the Beijing-Tianjin-Hebei patent cooperation network. Compared with the pair of Beijing-Tianjin and the pair of Beijing-Hebei, Tianjin and Hebei were not closely connected. In the future, Beijing-Tianjin-Hebei collaborative innovation should strengthen cooperation between Tianjin and Hebei.
\end{abstract}

\section{Introduction}

Beijing-Tianjin-Hebei (BTH) integration plan is an important national strategy aimed for the sustainable development of the BTH Urban Agglomeration. In the past, Beijing as the capital of China abstracted the best talent, technology, and capital from all over the country while the region surrounding Beijing is somewhat overshadowed. As the unique first-tier city in the North of China, Beijing plays a role of siphonage on the region surrounding Beijing through the spillover effect [1]. Unlike the balanced development in Yangtze River Delta, the region surrounding Beijing has significantly lagging economic development levels. However, BTH integration plan will change it and aims to create the third growth pole of the Chinese economy after the Yangtze River Delta and Pearl River Delta.

Nowadays, innovation has become an important driving force for economic development. Accordingly, collaborative innovation plays an important role in the coordinated development of BTH. In recent years, some scholars have explored the cooperative innovation in BTH Urban Agglomeration from the perspective of network based on 
copatenting, copublications, or patent right transaction data. Liu et al. [2] portrayed the spatial dynamics of intercity technology transfer networks in China's three urban agglomerations based on patent right transaction data from 2008 to 2015. Chen et al. [3] explored the evolution of regional innovation ability based on the innovation network constructed by using the cooperation patent data of " 211 universities" in Beijing, Tianjin, and Hebei regions during 2002-2016. Shi et al. [4] portrayed the scientific cooperation network of Chinese scientists and the spatial distribution characteristics. Lyu et al. [5] revealed the spatial organization and evolution characteristics of the innovation network of BTH Urban Agglomeration based on coauthorized patent data. Xing and Zhang [6] observed the innovation network evolution process of BTH Urban Agglomeration and compared the network features with Yangtze River Delta Urban Agglomeration based on the copatenting data of the World Intellectual Property Organization. The previous studies have studied the network characteristics and spatial distribution characteristics of the innovation network in BTH Urban Agglomeration constructed at the city level or microlevel. In this paper, we focused on the community structure in the innovation network of BTH Urban Agglomeration.

With the rapid development of technology, in some fields with dispersed knowledge, no one firm can lead the position in all aspects. It is difficult to create significant innovations for the entire market on its own. In this situation, firms have to seek collaboration from the outside world. Hence, the innovation cooperative network forms. In this complex network, the community structure raised the attention of Coakes and Smith [7]. They termed this kind of community structure as a community of innovation and deemed it as a special case of the more general community of practice dedicated to the support of innovation. Lim and Ong [8] further clarified the relationship between the community of innovation and community of practice. Yi and $\mathrm{Li}$ [9] focused on the mechanism of knowledge transfer between enterprises. Researchers have formed a consensus that a community of innovation plays a vital role in the innovation generation $[10,11]$. However, the empirical research is still very rare. The current research mainly focuses on the theoretical level although Coakes and Smith had pointed out that network visualization and analysis (NVA) and social network analysis (SNA) were feasible means to detect the relationships between organizational actors and map their social networks. One of the reasons is that the empirical data of innovation cooperative networks is not available. With the availability of data and the development of methodology, researchers can detect and visualize communities of innovation in innovation cooperative networks. Gloor et al. [12] visualized the communication patterns in cooperative innovation networks constructed on e-mail archives and selected three communities. Smith [13] visualized a network map showing who goes to whom for specific information in a target community. However, these communities visualized are usually on a small scale and the community detection technique has no necessity of use in the research.
In recent years, community detection techniques have been used in the detection of technology clusters or scientific knowledge clusters based on the network of patent or scientific publications [14-17]. The community detected in the citation network of patent or scientific publications is different from the community of innovation. Community of innovation consists of innovators and their cooperation relationship; however, in the patent citation network, the nodes are patents or scientific publications and the links are the citations. The aim of detecting the community of innovation is to find who cooperates with whom and what characterizes the community; however, the aim of detecting technology clusters is to explore the technological change and predict the emerging technologies.

This paper detected and visualized the communities of innovation in BTH Urban Agglomeration based on the patent cooperation network by using community detection techniques in SNA. The patent cooperation network was constructed with organizational innovators and their patent cooperative relationship. Patent is an important form of innovation output; therefore, we use the patent cooperation network to portray innovation cooperative networks. Through community detection, we hope to have a deeper understanding of the innovation cooperative network in BTH Urban Agglomeration. From the communities of innovation, we can find the features of the innovation cooperation in BTH Urban Agglomeration.

\section{Materials and Methods}

\subsection{Data}

2.1.1. Dataset. Three data sources were used in this paper. The first was China Patent Database from the State Intellectual Property Office (SIPO) from 1985 to March 2018, which contained 19 variables including patent application time, inventor, patentee, and patent category. The second was Business Registration Database (BRD) from the State Administration of Industry and Commerce (SAIC) which contained 15 variables including enterprise name, province, city, district, 4-digit industry code, registered capital, foundation date, and address. The third was data from the TianYanCha Database (TYCD), which collected information on more than 180 million social entities (including enterprises, institutions, foundations, schools, and law firms) in the country, including listing information, enterprise background, enterprise development, judicial risk, business risk, business status, and intellectual property rights.

2.1.2. Data Processing. Data processing and matching was essential in this study and there were five steps to construct the patent cooperation network. First, we filtered out those patents that had only one patentee. Second, we split the multiple patentees into array and each patentee was the element of the array. Third, we filtered out the individual patentees because they were not valid organizations. We identified an organization as valid only if its name was contained in BRD or TYCD. BRD contained all business registration information except nonprofit organizations 
such as universities and research institutions. As supplementary, we used TYCD that contained information about nonprofit organizations. Forth, we constructed the data frame of patent cooperation network by reshaping the patentee array. For example, if a patent contained three organization patentees, we wrote the array as $\left[p_{1}, p_{2}, p_{3}\right]$, where each element stood for an organization patentee. We reshaped the array into three arrays, that is, $\left[p_{1}, p_{2}\right]$, $\left[p_{1}, p_{3}\right]$, and $\left[p_{2}, p_{3}\right]$. The pair of elements in the newly reshaped arrays constituted two nodes and one edge between them in the patent cooperation network, meaning that the two organization patentees had patent cooperation relationship. Finally, we extracted the edges with both nodes belonging to BTH Urban Agglomeration. Since BRD and TYCD supplied the geographical information of all the organizations, we could easily filter out the nodes and edges that did not belong to BTH Urban Agglomeration.

From 1985 to March 2018, SIPO received 21.7 million patent applications, $4.4 \%$ of which were cooperative patents between organizations. There were 125 thousand organizations in the patent cooperation network, of which 57\% were domestic enterprises, $27 \%$ were universities and institutions, and $16 \%$ were foreign organizations. Before 2000, only few organizations cooperated in patent applications while the number has grown rapidly since then. We extracted 128 thousand cooperation relations and 9643 organizations for the patent cooperation network of BTH Urban Agglomeration from the national network. Within all the organizations which had a patent cooperation relationship in BTH Urban Agglomeration, 6093 organizations were from Beijing, 1838 from Tianjin, and 1712 from Hebei, accounting for $63.2 \%, 19.1 \%$, and $17.8 \%$, respectively. Therefore, it can be seen that Beijing played a key role in the patent cooperation network of BTH Urban Agglomeration.

2.2. Methodology. Social network analysis is a multidisciplinary emerging field based on graph theory and using statistical, computer, and visualization techniques as analysis methods. Since the later 20th century, this method has received much attention from scholars in many disciplines such as physics, mathematics, biology, sociology, and economics.

Network can be defined as a graph $G:=(V, E) . V$ is the collection of nodes representing cooperative organizations such as enterprise, university, and research institution. $E$ is the collection of edges representing patent cooperative relationship with weight $W$. The more frequently they collaborate in patent application, the larger the weight is. Larger weight means closer connection. Since collaboration has no direction, we deem the patent cooperation network as an undirected graph.

2.2.1. Indicator Based on Network Description. Many quantitative indicators have been defined on networks. In this paper, we applied the following indicators to describe the innovation cooperation network of BTH Urban Agglomeration:
(1). Community Size. Community size refers to the number of nodes contained in the community.

(2). Degree Centrality. Degree centrality is the simplest measure of centrality, which measures how many links a node has. In a given graph $G:=(V, E)$ with $n$ vertices and $m$ edges, the degree centrality of a vertex $v$ is defined as

$$
C_{D}(v)=\operatorname{deg}(v) .
$$

(3). Cooperative Intensity. In order to measure how many different cooperators an organizational innovator has, we defined cooperative intensity. Let $L$ denote the number of edges without weight in graph $G$. Cooperate intensity is as follows:

$$
\text { cooperative intensity }=\frac{2 L}{n} \text {. }
$$

2.3. Community Detection Technique. Community refers to a group of nodes with dense connections within the group and sparser connections between the groups. Community detection is crucial for the understanding of the internal organizations of complex networks. Over the last decades, community detection has become one of the most popular subfields in social network analysis. Many community detection methods have been developed and applied in a variety of fields such as sociology, biology, and statistics. Fast Greedy algorithm [18], Walktrap [19], Infomap [20], Label Propagation [21], Multilevel [22], and Spinglass [23] are the most popular and widely used algorithms in the field of community detection. Some research $[24,25]$ compared these algorithms and showed that Spinglass, Multilevel, and Fast Greedy performed better than other algorithms. Further, Spinglass performed more robust on community detection regarding perturbations. In this paper, we applied Spinglass community detection algorithm to identify the communities in the innovation cooperation network of BTH Urban Agglomeration. Spinglass algorithm employs a Spinglass model from statistical physics to provide a rigorous foundation for combining external knowledge into community detection processes [26]. It is a semisupervised community detection algorithm. The goal of Spinglass algorithm is not to maximize the "modularity" which is a global objective measure of how good the global community structure discovered by an algorithm is [27]. It can both obtain high-scoring modularity and meet the provided requirement.

We compared five community detection algorithms to find the best one to fit the BTH cooperative network. The summarized results (Table 1) showed that Fast Greedy and Spinglass had the highest modularity. Different algorithms varied greatly in the number of communities and the community size. Walktrap and Label Propagation had more than 500 communities with the highest coefficient of variation $(\mathrm{CV})$ of community size. Although the Fast Greedy algorithm had 0.83 modularity, it generated too many communities with extremely small size. There were only 15 
TABLE 1: Comparison of community detection algorithms in BTH patent cooperation network.

\begin{tabular}{lccccc}
\hline Algorithm & Fast Greedy & Walktrap & Label Propagation & Multilevel & Spinglass \\
\hline Modularity & 0.83 & 0.75 & 0.73 & 516 & 0.81 \\
Number of communities & 115 & 531 & 934 & 57 & 0.83 \\
Maximal community size & 1300 & 758 & 12 & 774 & 784 \\
Mean of community size & 55 & 12 & 2 & 111 & 327 \\
Minimal community size & 2 & 2 & 3.8 & 318 \\
CV of community size & 2.85 & 4.3 & & 1.31 & 0.29 \\
\hline
\end{tabular}

communities with more than 100 nodes and more than $71 \%$ of the communities were with less than 20 nodes. Therefore, in order to better understand the structure of the BTH patent cooperation network, we finally chose the Spinglass algorithm.

\section{Results}

3.1. Characteristic of Patent Cooperation Network. We extracted organizations of BTH Urban Agglomeration from the entire national network and constructed an innovation cooperation network of BTH Urban Agglomeration with 9643 nodes (Figure 1). In the network, the largest connected subgraph contained 6319 nodes accounting for $66 \%$ of the total. Except the largest connected subgraph, the other 1370 subgraphs were all on a small scale; most of them consisted of two nodes.

Visualizing the largest connected subgraph (Figure 2), we found that Beijing organizations (yellow nodes) dominated the patent cooperation network of BTH Urban Agglomeration. Within the top 30 organizations, 24 (80\%) were from Beijing and within the top 10 organizations, and 9 (90\%) were from Beijing. From the perspective of the organizational category, universities and some state-owned enterprises occupied the center of the network and acted as bridges (Table 2).

3.2. Communities of Innovation. Except the largest connected subgraph, other subgraphs were on a small scale, most of which consisted of only two nodes. Therefore, we only detected communities in the largest connected subgraph with the aim of exploring the modes of patent collaboration in BTH Urban Agglomeration.

Since the frequency of collaboration obeyed power-law distribution with few organizations collaborating frequently while the others rarely, we took logarithm transformation of the frequency in order to eliminate the huge difference. We designated the number of communities in Spinglass algorithm from 6 to 25, since too many or too few communities did not benefit the understanding of network structure. By comparing the modularity under different designated numbers of communities, ultimately we chose 12 communities of innovation (Figure 3 ) by using Spinglass algorithm in $R$ package igraph.

We further used a hierarchical cluster algorithm to cluster 12 communities into clusters based on six clustering variables reflecting the structure characteristic of each community (Table 3 ). We standardized the six clustering

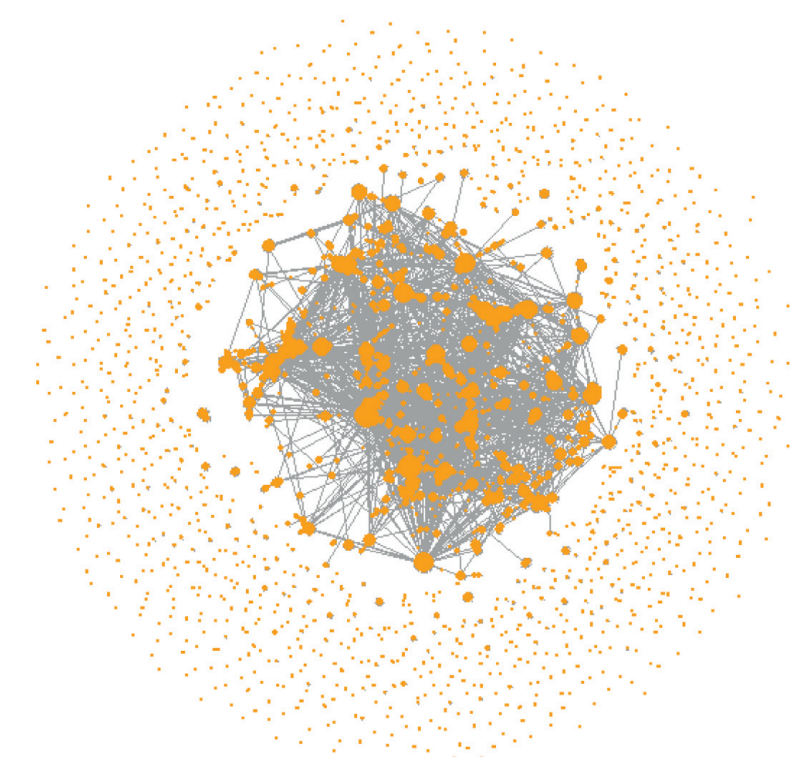

Figure 1: Patent cooperation network in BTH Urban Agglomeration.

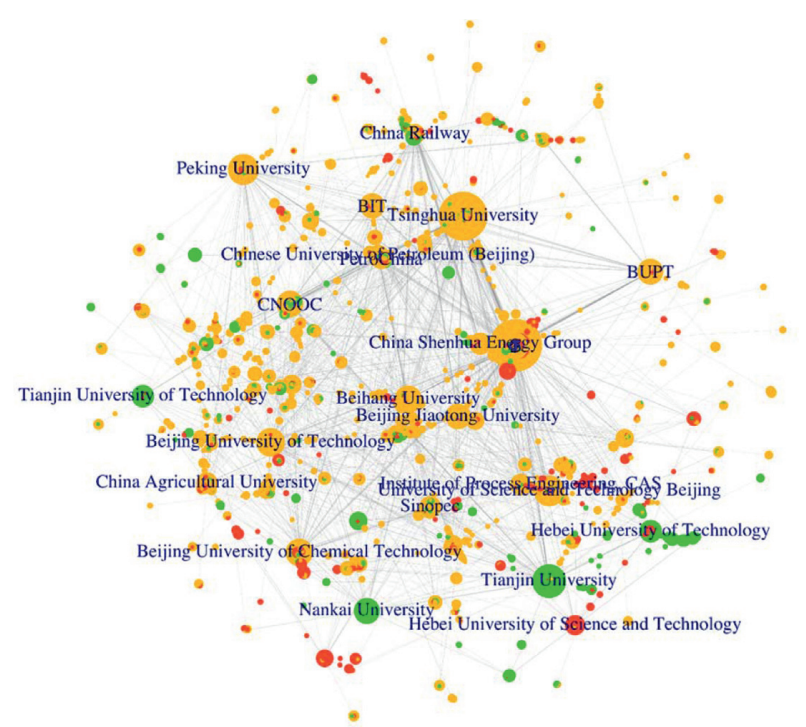

FIgURE 2: Visualization of the largest connect subgraph. Note: yellow nodes represent Beijing organizations, red nodes represent Hebei organizations, and green nodes represent Tianjin organizations.

variables and used ward.D2 cluster method based on Euclidean distance in the hierarchical cluster algorithm. Elbow method was used to determine the optimal number of 
TABLE 2: The top 20 organizations with the highest degree centrality in BTH Urban Agglomeration.

\begin{tabular}{|c|c|c|c|c|c|}
\hline Rank & Name & Centrality & Rank & Name & Centrality \\
\hline 1 & State Grid Corporation & 685 & 11 & China National Offshore Oil Corporation & 125 \\
\hline 2 & Tsinghua University & 575 & 12 & $\begin{array}{l}\text { Beijing University of Posts and Telecommunications } \\
\text { (BUPT) }\end{array}$ & 117 \\
\hline 3 & Tianjin University & 225 & 13 & North China Electric Power University & 116 \\
\hline 4 & Peking University & 183 & 14 & Nankai University & 116 \\
\hline 5 & $\begin{array}{c}\text { University of Science and Technology } \\
\text { Beijing }\end{array}$ & 181 & 15 & Beijing Jiaotong University & 112 \\
\hline 6 & Beijing University of Technology & 150 & 16 & Beijing Institute of Technology (BIT) & 110 \\
\hline 7 & China Electric Power Research Institute & 146 & 17 & Hebei University of Technology & 82 \\
\hline 8 & Beihang University & 136 & 18 & State Grid Electric Power Company, Beijing & 81 \\
\hline 9 & $\begin{array}{l}\text { Beijing University of Chemical } \\
\text { Technology }\end{array}$ & 135 & 19 & Tianjin University of Science and Technology & 81 \\
\hline 10 & $\begin{array}{c}\text { State Grid Power Company in Hebei } \\
\text { Province }\end{array}$ & 126 & 20 & China Shenhua Energy Group & 79 \\
\hline
\end{tabular}

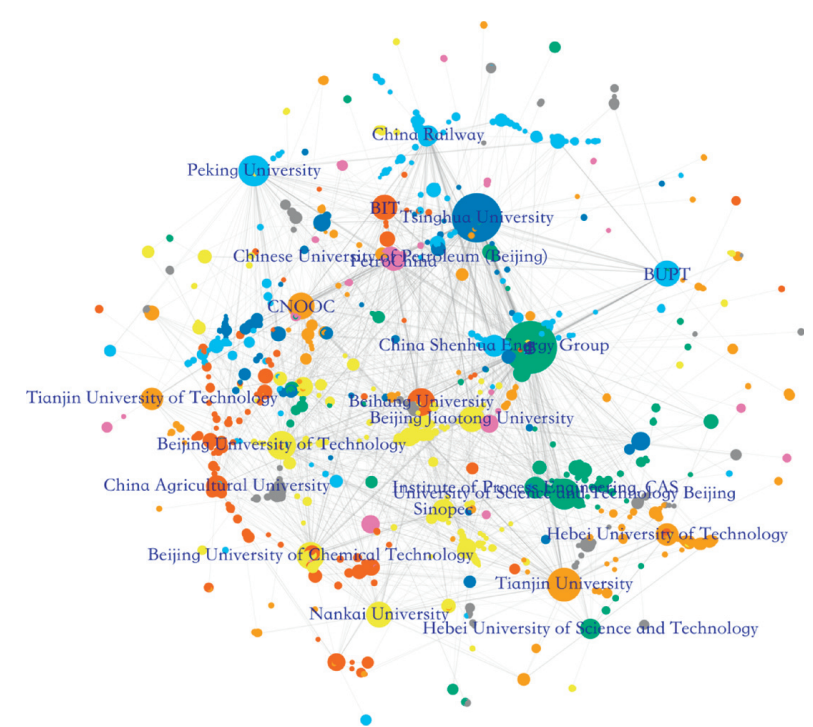

FIGURE 3: Visualization of the 12 communities of innovation. Note: different colors denote different communities of innovation.

TABLE 3: Clustering variables and description.

\begin{tabular}{lc}
\hline Variable & Description \\
centr $_{1 s t}$ & The proportion of the highest degree centrality \\
ratio $_{12}$ & The ratio of the highest degree centrality to the second high \\
Ratio $_{13}$ & The ratio of the highest degree centrality to the third high \\
ratio $_{14}$ & The ratio of the highest degree centrality to the fourth high \\
Ratio $_{15}$ & The ratio of the first highest degree centrality to the fifth high \\
coop-int & The number of different cooperators an organizational innovator has \\
\hline
\end{tabular}

clusters. Figure 4 shows that the optimal number of clusters was four where the curve became flat.

Table 4 shows the group members of each cluster and their features. The mean of cooperative intensity is ranked as cluster $\# 1>$ cluster $\# 4>$ cluster $\# 3>$ cluster $\# 2$. Except for cooperative intensity, the mean of the other five variables is ranked as \# $2>$ cluster \# $1>$ cluster \#3 > cluster \#4. Furthermore, analysis of variance (ANOVA) showed a significant difference in the four clusters given a significant level of 0.05 .

\section{Discussion}

Sort the 12 communities according to their size in descending order and number them no. 1-12. We will discuss the structural characteristics and industrial 


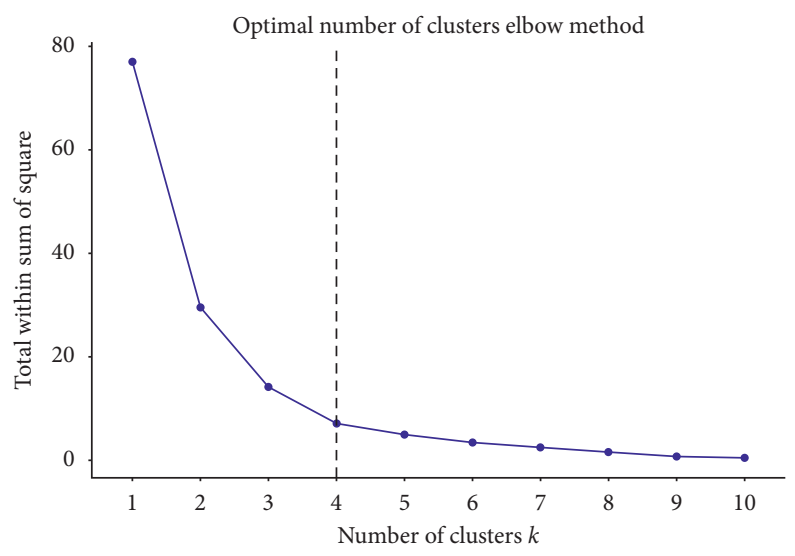

FIgURE 4: Plot of the total within the sum of the square with the number of clusters.

TABLE 4: Group members of each cluster and their features.

\begin{tabular}{|c|c|c|c|c|c|c|c|}
\hline $\begin{array}{l}\text { Cluster } \\
\text { no. }\end{array}$ & Community no. & $\begin{array}{c}\text { Mean of centr } r_{1 s t} \\
(\%)\end{array}$ & $\begin{array}{l}\text { Mean of } \\
\text { ratio }_{12}\end{array}$ & $\begin{array}{l}\text { Mean of } \\
\text { ratio }_{13}\end{array}$ & $\begin{array}{c}\text { Mean of } \\
\text { ratio }_{14}\end{array}$ & $\begin{array}{c}\text { Mean of } \\
\text { ratio }_{15}\end{array}$ & $\begin{array}{l}\text { Mean of } \\
\text { coop-int }\end{array}$ \\
\hline 1 & 1 & 16 & 4.69 & 5.44 & 5.91 & 8.46 & 4.86 \\
\hline 2 & 2 & 27 & 11.27 & 14.02 & 19.83 & 21.30 & 2.32 \\
\hline 3 & $3,4,5$, and 7 & 11 & 1.76 & 3.36 & 4.69 & 5.80 & 2.52 \\
\hline 4 & $6,8,9,10,11$, and 12 & 7 & 1.45 & 2.02 & 2.45 & 2.89 & 2.88 \\
\hline
\end{tabular}

characteristics of these 12 communities of innovation in BTH Urban Agglomeration in the following.

4.1. Structural Characteristic of Communities. According to the features of the four clusters, we summarized four typical structures of the community: hierarchical structure, singlecenter structure, polycentric structure, and flat structure. In the following, we will illustrate these four structures in detail.

4.1.1. Hierarchical Structure. Hierarchical structure consists of first-level central nodes, second-level central nodes, and slave nodes. The first-level central node takes the central position. The second-level central node surrounds the firstlevel central node and at the same time acts as the central node itself with other slave nodes surrounding it. Cluster \#1, which only contains community \#1, is a typical hierarchical structure. Community \#1 is the largest community of innovation with 784 nodes mainly composed of universities, research institutes, and enterprises related to electricity and power grid. Therefore, we named it as the Electric Power Community of Innovation (Figure 5).

In hierarchical structure, the first-level central node usually accounted for a high proportion of degrees, and the degree ratios of the first-level central node to the secondlevel central nodes were high too. This feature made the structure hierarchical. Besides, we speculated that hierarchical structure encouraged more connections with different nodes since it had the highest cooperative intensity compared with the other three structures.

The first-level central node in the Electric Power Community of Innovation was the State Grid Corporate and the second-level central nodes were the China Electric Power Academy, State Grid Power Company in Hebei Province, North China Electric Power University, and State Grid Power Company in Beijing. Due to the high-tech and highcapital characteristics of the power industry, innovation cooperation in the power industry was more necessary. Meanwhile, the monopoly nature of the power industry was obvious. State Grid Corporate, the head enterprise, inevitably occupied the most important position. Most of the organizations in the Electric Power Community had close relationships with the State Grid Corporation in the form of subsidiaries, joint-stock, common industry filed, and so on, which cleared the barriers to innovation cooperation and made it the biggest community in BTH Urban Agglomeration. The number of cooperative patents in the Electric Power Community was 58 thousand accounting for $32 \%$ of the largest connected subgraph and $29 \%$ of the entire network.

From the geographical characteristics (Table 5), community \#1 had the highest proportion of organizations from Hebei Province, accounting for 36\%. The geographical characteristics of community \#1 indicated that in the field of electric power Hebei Province had a close relationship with Beijing while it had a weak relationship with Tianjin. Within all the connections between regions in community \#1, the proportions of connections between Beijing-Hebei, BeijingTianjin, and Hebei-Tianjin were $76.1 \%, 22.3 \%$, and $1.6 \%$, respectively. This characteristic was also true for community \# 4, which was in the field of metallurgy and materials.

Actually, from the view of the whole network, we could find that there was no close relationship between Tianjin and Hebei. Strong relationships only existed between Beijing and 


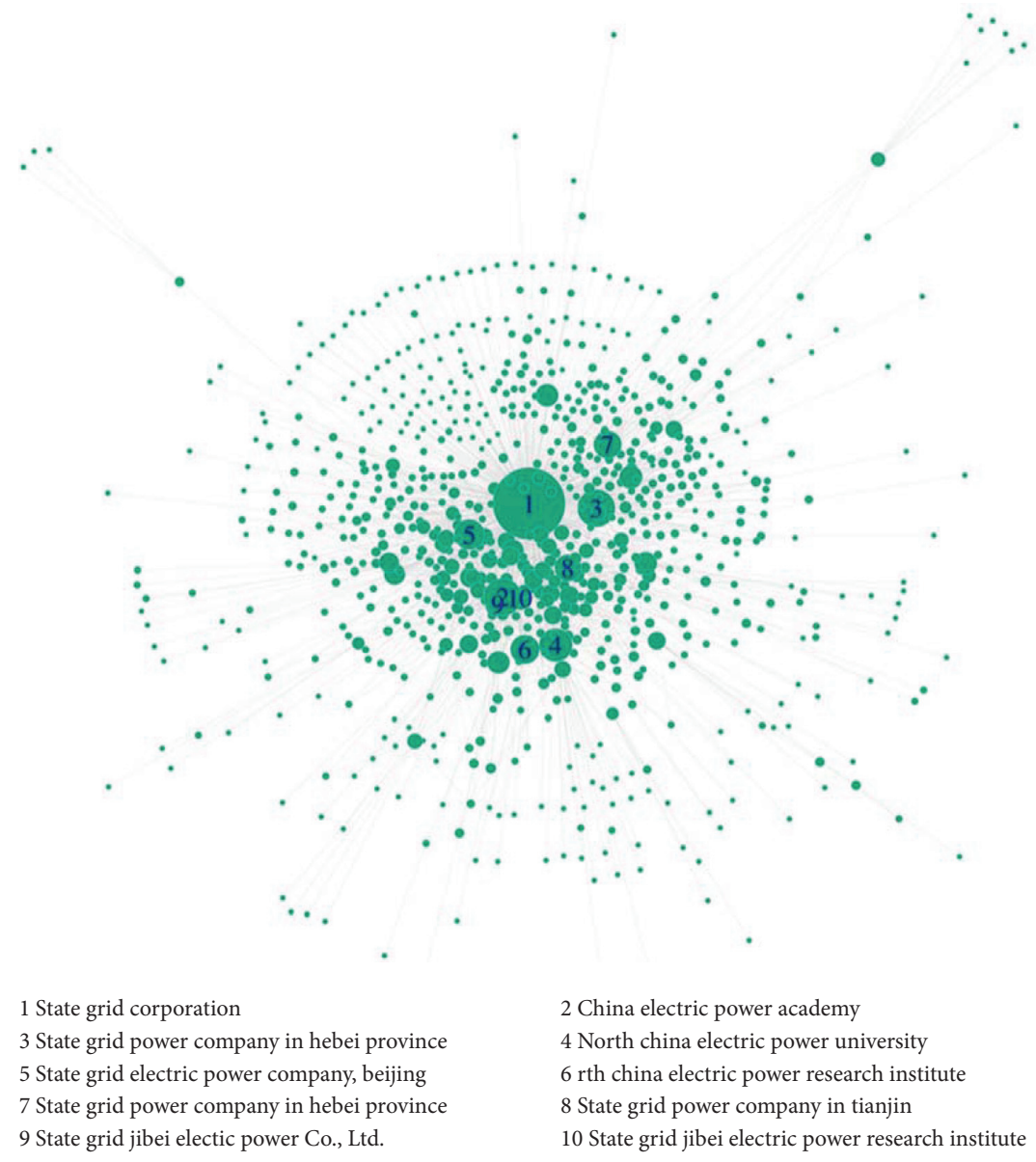

Figure 5: Electric Power Community of Innovation with hierarchical structure.

TABLe 5: Geographical characteristics of communities of innovation in BTH Urban Agglomeration.

\begin{tabular}{lccc}
\hline Community no. & Beijing (\%) & Tianjin (\%) & Hebei (\%) \\
\hline 1 & 54 & 9 & 36 \\
2 & 86 & 7 & 7 \\
3 & 71 & 7 & 22 \\
4 & 63 & 7 & 30 \\
5 & 31 & 59 & 11 \\
6 & 80 & 10 & 9 \\
7 & 86 & 4 & 10 \\
8 & 56 & 31 & 13 \\
9 & 62 & 17 & 22 \\
10 & 40 & 49 & 11 \\
11 & 78 & 11 & 12 \\
12 & 59 & 21 & 19 \\
\hline
\end{tabular}

Tianjin or between Beijing and Hebei. To be more specific, there were 1712 nodes in the $\mathrm{BTH}$ network belonging to Hebei Province with 2797 edges, within which, 38\% were connected inside Hebei and 53\% connected Beijing and Hebei, while only $9 \%$ connected Tianjin and Hebei. For Tianjin, there were 1838 nodes in the BTH network with 2812 edges, within which, $53 \%$ were connected inside Tianjin and 38\% connected Beijing and Tianjin, while only $9 \%$ connected Tianjin and Hebei. As for Beijing, 6093 nodes in the BTH network belonged to Beijing with 10038 edges, within which, $74 \%$ were connected inside Beijing, $11 \%$ connected Beijing and Tianjin, and 15\% connected Beijing and Hebei. It was obvious that Beijing dominated the BTH network and connected Tianjin and Hebei, respectively. It reflected the lack of innovative connections between Tianjin and Hebei.

Some of the communities have significant geographical attributes. For example, more than $80 \%$ of organizations of communities \#2, \#6, and \#7 were from Beijing, and about half of the organizations of communities \#5 and \#10 were from Tianjin. This phenomenon reflected geographical agglomeration. We speculated that geographical proximity facilitated patent cooperation.

4.1.2. Single-Center Structure. Single-center structure community consisted of one single node as core and the other nodes surrounding it. The single node occupied the dominant position and the others were subordinate status. Cluster \#2, which only contained community \#2, was a typical single-center structure. Since the center of community \#2 was Tsinghua University, we named it as Tsinghua Community of Innovation (Figure 6). Singlecenter structure had two features. First, it had a single central node with very large degree centrality and other nodes with much smaller degree centrality. Second, due to 


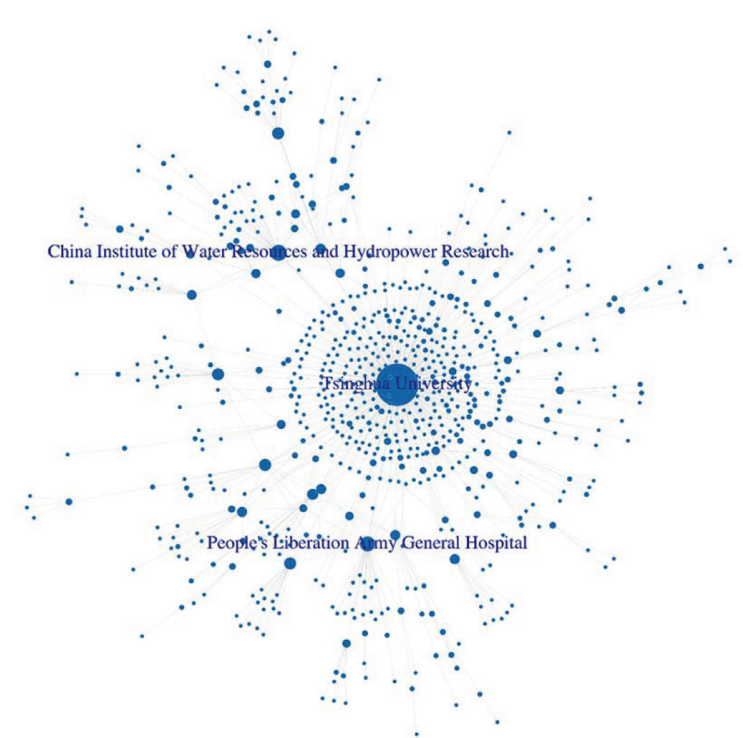

Figure 6: Tsinghua Community of Innovation with single-center structure.

the lack of connection between other nodes, the network connection is not dense. From Table 3, we can see in Tsinghua Community of Innovation that centr ${ }_{1 s t}$ was the highest and ratio ${ }_{12}$, ratio $_{13}$, ratio $_{14}$, and ratio ${ }_{15}$ were the highest too. Nevertheless, the cooperative intensity was the lowest in the four structures, which indicated that the single-center structure was not conducive to generating innovative links.

Usually, the central node in a single enter structure should meet two characteristics. The first is depth, which requires strong $R \& D$ capability to become an ideal partner of other organizations. The second is breath, which requires broad research fields to intersect with other organizations in different fields and to form complementary technologies. Only comprehensive universities or large enterprises can meet these two characteristics. In the Tsinghua Community of Innovation, Tsinghua University acts as the central node with strong R\&D capability and broad research fields connecting with other organizations. Tsinghua University dominates this community and other organizations' layout around it. The degree centrality of Tsinghua University is 575, more than 10 times of the China Institute of Water Resources and Hydropower Research, whose centrality is the second largest in this community. Meanwhile, the betweenness centrality of Tsinghua University is 8 times of the second largest node. It indicates that the cooperative relationship in the community is very dependent on the central node. Due to the lack of direct connection between each other, they need to connect through the central node, which means the central node occupies the structural hole in the network. According to the structural hole theory developed by Burt [28], the node on the structural hole can gain an important comparative advantage.

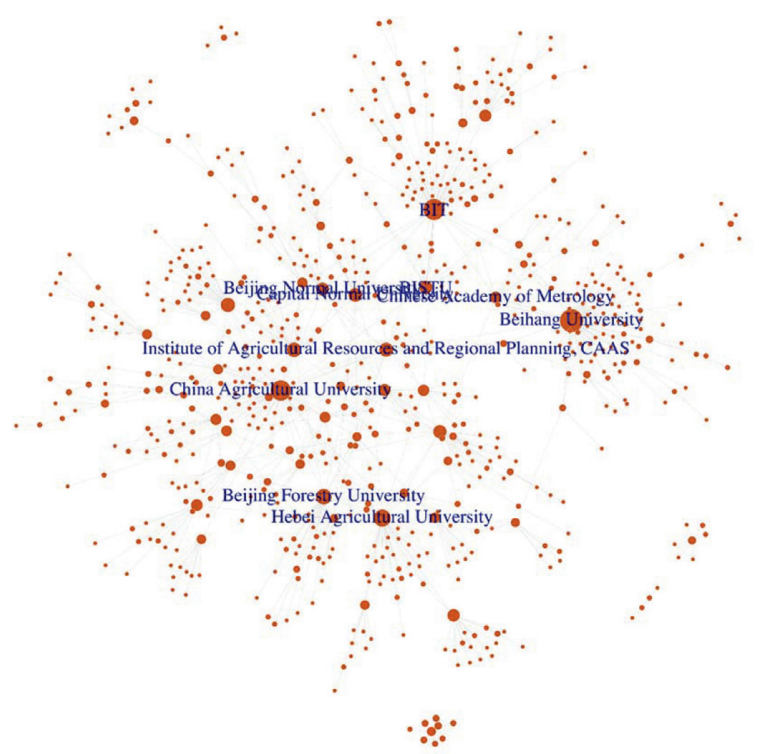

FIGURE 7: Community of innovation with multiple centers of Beihang University, Beijing Institute of Technology, and China Agricultural University.

4.1.3. Polycentric Structure. Polycentric structure community has multiple central nodes whose degree centrality is even. Cluster \#3, which consisted of communities \#3, \#4, \#5, and \#7, was typical polycentric structures. In the following, we will illustrate communities \#3 and \#7 as examples to show the polycentric structure.

Community \#3 (Figure 7) had three central nodes that were Beihang University, BIT, and China Agricultural University accounting for $7 \%, 5 \%$, and $4 \%$ of the total degree centrality, respectively. Beihang and BIT were local centers with many subordinate nodes surrounding them. Their structures were very like single-center structures but on a small scale. The subcommunity of China Agricultural University had a hierarchical structure with China Agricultural University as the first-level central node and Hebei Agricultural University and Beijing Forestry University as second-level central nodes forming a community of innovation in agricultural fields.

We found that the three multiple centers were not connected with each other directly but connected via an important node that was Institute of Agricultural Resources and Regional Planning, Chinese Academy of Agricultural Sciences (CAAS). The shortest paths between BIT, Beihang University, and China Agricultural University all passed this node.

Community \#7 (Figure 8) was also a polycentric structure with Peking University and BUPT as centers whose proportions of degree centrality were $12 \%$ and $8 \%$, respectively. Besides, there were several important organizational innovators with $2 \%$ proportion of degree centrality, such as China Mobile Communications Corporation (CMCC), Institute of Computing Technology of Chinese Academy of Sciences, and Beijing Nonferrous Metal 


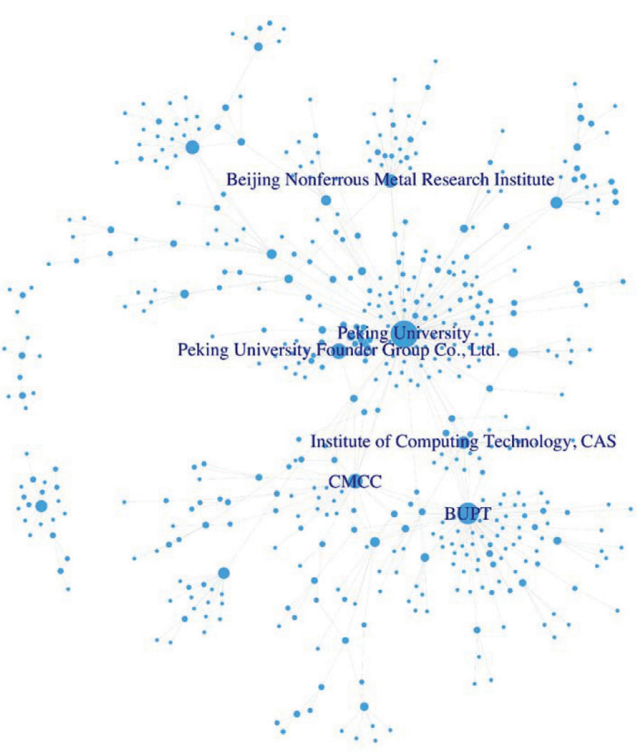

Figure 8: Community of Innovation with multiple centers of Peking University and BUPT.

Research Institute. Unlike the polycentric community of Beihang, BIT, and China Agricultural University, the multiple centers, Peking University and BUPT, had connections directly; namely, they had patent collaboration.

Compared with single-center structure, the proportion of the highest degree centrality in polycentric structure was relatively lower but the multiple centers had relatively uniform degree centrality.

4.1.4. Flat Structure. Flat structure had no obvious central nodes. Cluster \#4, which contains six communities, was a typical flat structure. In cluster \#4, the mean of centr $_{1 s t}$ was the lowest and the means of ratio $o_{12}$, ratio $_{13}$, ratio $_{14}$, and ratio $_{15}$ were the lowest too, which meant the distribution of the degree centrality was uniformly in flat structure.

Flat structure was similar to polycentric structure. From Figure 9, we can see that cluster \#4 whose structure was flat had the shortest distance to cluster \#3 whose structure was polycentric. However, different from the polycentric structure, the top largest nodes in flat structure had not so large degree centrality. In the following, we will illustrate community \#11 as an example to show flat structure.

Community \# 11 (Figure 10) was mainly in the field of the construction industry. The top three nodes with the highest degree centrality were China Construction First Bureau Group, China Construction Corporation, and China Academy of Building Research, whose degree centrality accounted for $3 \%, 3 \%$, and 3\%, respectively. Different from the above three types of structure, flat structure had no obvious central nodes in the network. However, the cooperation intensity was higher than the single-center structure and polycentric structure. Flat structure was usually on a small scale. In this study, communities \#6, \#8, \#9, \#10, \#11, and \#12 had flat structure which were small communities while hierarchical structures usually were on a large scale. Due to fewer organizations, flat structure had less of a need

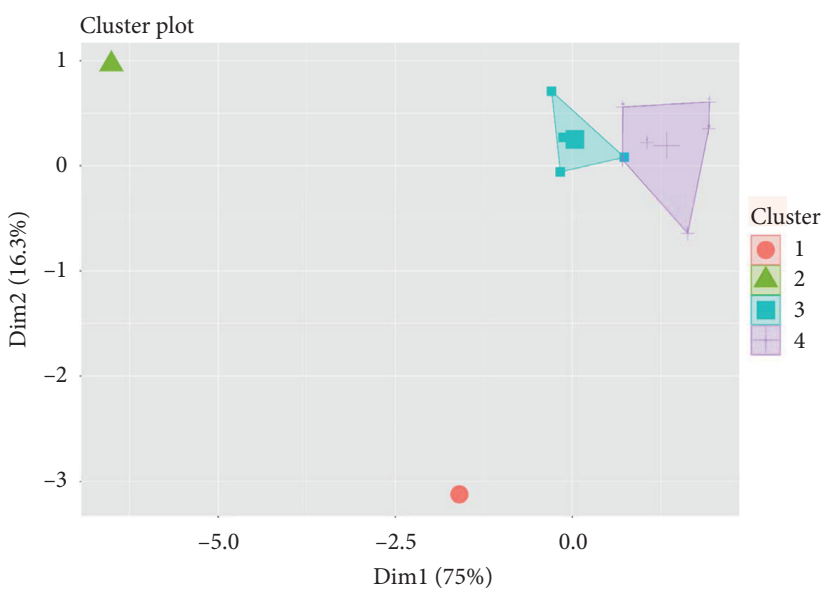

FIgURE 9: Cluster plot of hierarchical cluster algorithm.

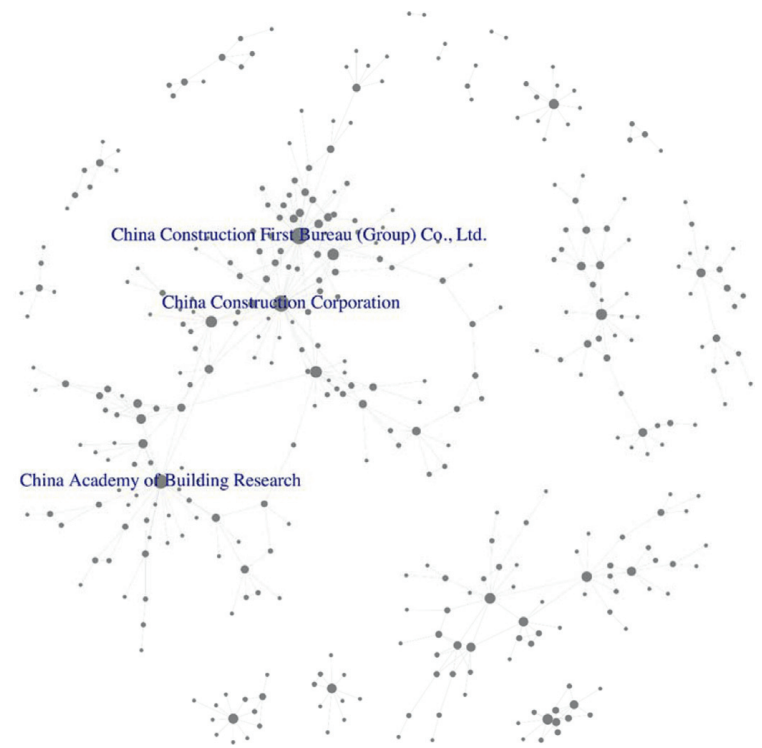

FIGURE 10: Community of construction industry with flat structure.

for hierarchical management. The degree centrality was evenly distributed throughout the flat structure network, which meant there exited no dominant node in the network. The organizations collaborated with each other through comparative technological complementarity. Flat structure was a relatively loose innovation community structure.

4.2. Industry Characteristic of Communities. For better understanding the BTH network and communities, we named each community by the industrial characteristics of the top ten nodes with the largest degree centrality. Since the node degree centrality was power-law distribution, the top ten nodes could represent the overall characteristics of each community. The BRD and TYCD provided us with the industrial category attributes for each enterprise. For research institutions and some universities, we could judge their main research fields from their names. Based on the industrial category attributes of enterprises and the main 
TABle 6: Detailed information on the communities of innovation with industrial characteristics.

\begin{tabular}{|c|c|c|c|c|}
\hline Community no. & Industry filed & Community structure & Community size & Cooperation intensity \\
\hline 1 & Electric power & Hierarchical & 784 & 4.86 \\
\hline 4 & Metallurgy and materials & Polycentric & 582 & 2.44 \\
\hline 5 & Petroleum & Polycentric & 559 & 2.75 \\
\hline 6 & Municipal transportation & Flat & 537 & 2.73 \\
\hline 8 & Petroleum & Flat & 489 & 2.73 \\
\hline 9 & Railway & Flat & 379 & 3.47 \\
\hline 11 & Construction & Flat & 365 & 2.68 \\
\hline 12 & Petroleum & Flat & 318 & 2.92 \\
\hline
\end{tabular}

research fields of research institutions and some universities, we labeled the communities if they showed significant industrial characteristics. Within the twelve communities of innovation, eight communities had significant industrial characteristics. Except the communities of innovation in the field of electric power (community \#1) and construction (community \#11) illustrated above, there were still six communities with significant industrial characteristics. They were communities $\# 4, \# 5, \# 6, \# 8$, \#9, and \#12. Table 6 shows the detailed information of the eight communities. The eight communities of innovation mainly gathered in six industries, which were electric power, metallurgy and materials, petroleum, municipal transportation, railway, and construction. From the view of community structure, five of them were flat structure, two were polycentric structure, and one was hierarchical structure. Many researches $[29,30]$ had shown that moderate technological distance was beneficial for innovation. Innovation had a higher chance to emerge in the same industry due to the moderate technological distance. Different organizations in the same industry took their advantage to cooperate with each other.

From the view of organization type, the communities of innovation are usually composed of universities, enterprises, and research institutes. Since universities had a wide range of research fields and they usually had fewer conflicts of interest with enterprises in the intellectual property rights, universities often cooperated with many other organizations in the network. In five of the eight communities, the node with the highest degree centrality was university. In further, we calculated the proportion of each type of organization in the eight communities (Table 7). Although only $1 \%-3 \%$ of organizations were universities in the communities, universities played an important role in the network with high degree centrality.

Actually, universities not only had high degree centrality themselves but also played an important role in connecting regions. We attempted to find the key nodes connecting regions, which had more potential for increasing the cooperation between regions. We extracted the nodes that connected different regions most and found that universities and state-owned enterprises played important roles in connecting among the regions. Table 8 shows the top 20 organizations connecting different regions with the largest degree across regions, which was defined as the number of connections between different regions. Within the 20 organizations, universities accounted for eight of them, indicating that universities were very important to connect organizations between different regions. Some researches $[26,32]$ also showed that universities had central positions in university-industry network acting as the disseminators of knowledge and technology. In addition, state-owned enterprises were more likely to cooperate with their affiliated companies.

Among the eight communities with significant industrial characteristics, communities \#9, \#1, \#5, \#12, and \#8, which were in the field of railway, electric power, and petroleum, had more connections between different regions. The proportions of connection across regions in the communities $\# 9$, \#1, \#5, \#12, and \#8 were $37.0 \%, 32.7 \%, 32.0 \%, 28.0 \%$, and $27.7 \%$, respectively, ranking higher than the other 7 communities. It indicated that BTH Urban Agglomeration had more connections in patent application in the field of railway, electric power, and petroleum.

In the following, we will illustrate the other four industries reflected from the communities of innovation.

4.3. Petroleum Industry. Communities $\# 5$, \#8, and $\# 12$ (Figures 11-13) were three separate communities of innovation in the field of the petroleum industry. They centered on China's three oil giants, CNOOC, Sinopec, and PetroChina, respectively. In community $\# 5$, CNOOC connected with Tianjin University and Tianjin University of Technology, acting as the three multiple centers. Community $\# 5$ had strong geographical attributes. Since CNOOC mainly engaged in offshore oil exploitation, most of the organizations (59\%) were from Tianjin. This was very different from the majority of communities with most of the organizations located in Beijing. Community \#8 had a flat structure with Sinopec as one of the most important nodes. This community was prominent in the chemical industry, which was consistent with the area of expertise of Sinopec. Community \#12 was also a flat structure with PetroChina as an important node. The other important nodes include the Chinese University of Petroleum (Beijing), Tiangong University, Shenhua Group, and Eastern Geophysical Exploration Co., Ltd. Community \#12 showed outstanding performance in oil exploration, which was also the area of expertise of PetroChina.

4.4. Metallurgy and Materials. Community \#4 (Figure 14) was mainly in the field of metallurgy and materials. Shougang Group, Hebei Iron and Steel Group, and their subsidiaries were important enterprise nodes in this community. Metallurgy and materials industry occupied a large proportion in 
TABLE 7: Industry-university-research structure of the eight communities of innovation.

\begin{tabular}{lcccc}
\hline Community no. & Industry & University (\%) & Enterprise (\%) & Research institute (\%) \\
\hline 1 & Electric power & 0.8 & 95.3 & 4.0 \\
4 & Metallurgy and materials & 3.4 & 89.2 & 7.4 \\
5 & Petroleum & 1.8 & 90.0 & 8.2 \\
6 & Transportation & 1.9 & 89.4 & 8.8 \\
8 & Petroleum & 2.7 & 87.7 & 9.6 \\
9 & Railway & 1.1 & 89.4 & 9.5 \\
11 & Construction & 0.8 & 91.0 & 8.2 \\
12 & Petroleum & 1.6 & 92.1 & 6.3 \\
\hline
\end{tabular}

TABLE 8: Top 20 nodes with the largest degree across regions.

\begin{tabular}{|c|c|c|c|c|}
\hline Organizations & Province & Degree across regions & Degree & Industry \\
\hline State Grid & Beijing & 308 & 685 & Power \\
\hline Tianjin University & Tianjin & 78 & 225 & University \\
\hline China National Offshore Oil Corporation & Beijing & 75 & 125 & Petroleum \\
\hline Tsinghua University & Beijing & 74 & 575 & University \\
\hline University of Science and Technology Beijing & Beijing & 40 & 181 & University \\
\hline State Grid Hebei Electric Power Company & Hebei & 38 & 126 & Power \\
\hline CNOOC Energy Development Co., Ltd. & Beijing & 37 & 50 & Petroleum \\
\hline Hebei University of Technology & Tianjin & 35 & 82 & University \\
\hline China National Petroleum Corporation & Beijing & 34 & 69 & Petroleum \\
\hline Beijing University of Chemical Technology & Beijing & 33 & 135 & University \\
\hline State Grid Tianjin Electric Power Company & Tianjin & 33 & 54 & Power \\
\hline China Electric Power Research Institute & Beijing & 30 & 146 & Institution \\
\hline The Third Railway Survey and Design Institute Group Co., Ltd. & Tianjin & 29 & 38 & Railway \\
\hline North China Electric Power University & Beijing & 28 & 116 & University \\
\hline Institute of Process Engineering, CAS & Beijing & 26 & 74 & Institution \\
\hline Nankai University & Tianjin & 25 & 116 & University \\
\hline Chinese Academy of Military Medical Sciences & Tianjin & 25 & 28 & Institution \\
\hline CNPC Bohai Petroleum Equipment Manufacturing Co., Ltd. & Tianjin & 24 & 30 & Petroleum \\
\hline China Shenhua Energy Co., Ltd. & Beijing & 23 & 79 & Mining \\
\hline North China Electric Power University (Baoding) & Hebei & 21 & 26 & University \\
\hline
\end{tabular}

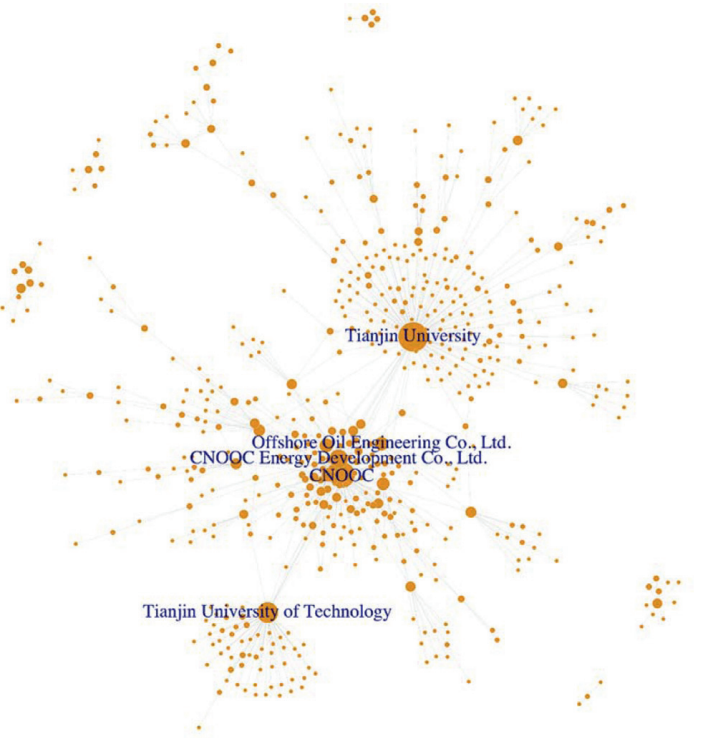

FIgURE 11: CNOOC community.

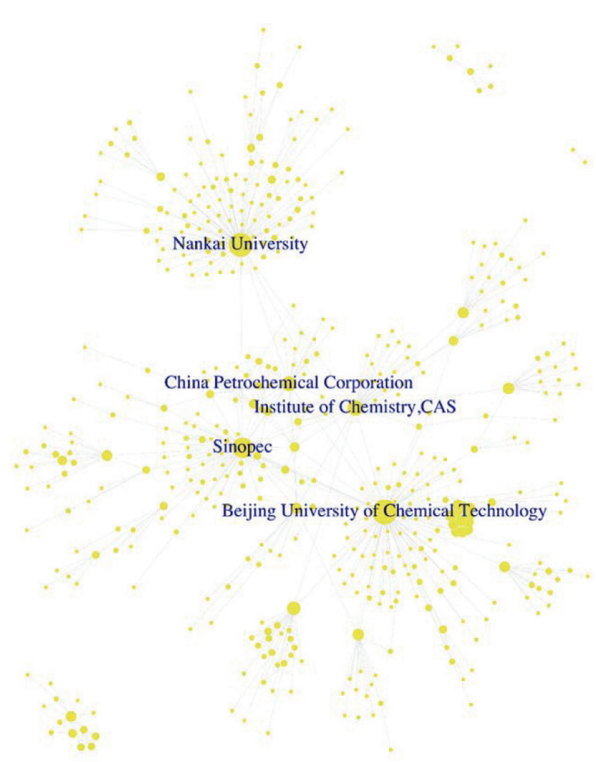

FIGURE 12: Sinopec community. 


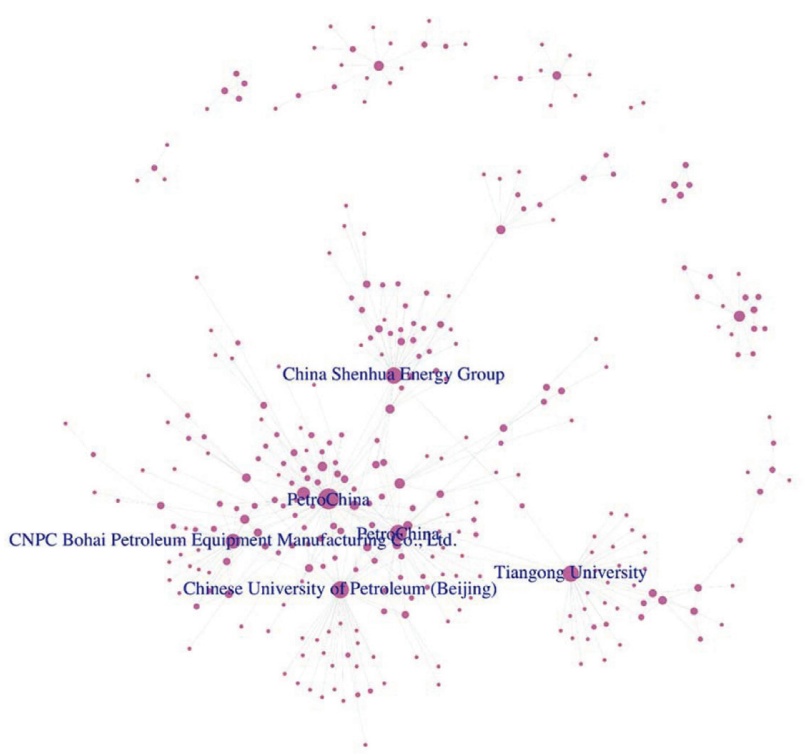

Figure 13: PetroChina community.

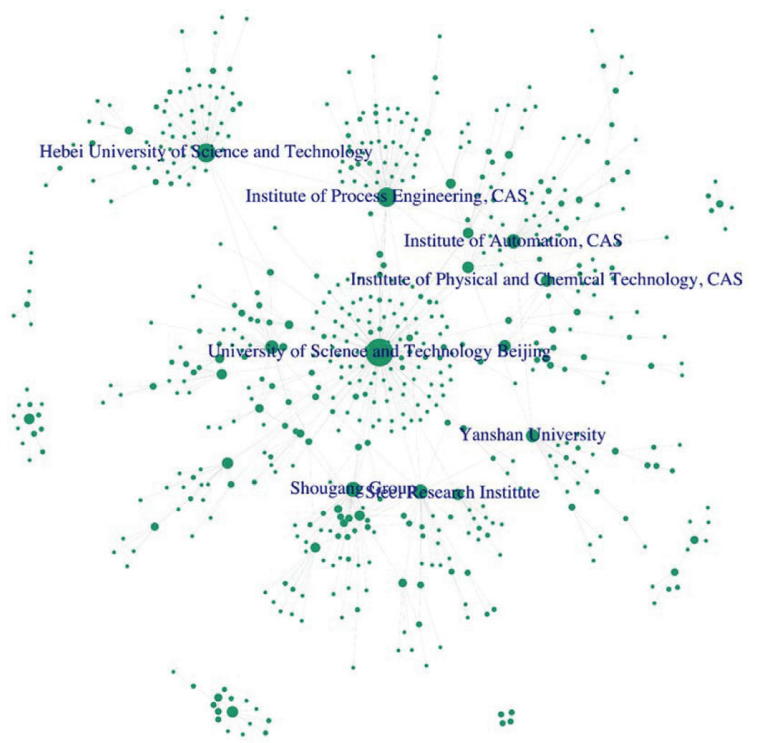

FIGURE 14: Community \#4 in the field of metallurgy and materials.

the industrial structure of BTH Urban Agglomeration, especially Hebei Province. Hebei Province was an important steel production base in China. Since 2002, Hebei has been the province with the largest steel output. In 2019, Hebei's steel output was 284 million tons, accounting for $23.6 \%$ of the country. Community \#4 had a close innovation cooperation relationship in BTH Urban Agglomeration. About 30\% of organizations in the community came from Hebei Province. Compared with the other communities, the proportion was relatively high, which was only lower than that of the Electric Power Community of Innovation.

4.5. Municipal Transportation. Community \#6 (Figure 15) was in the field of municipal transportation with flat structure. The first three important nodes in the community

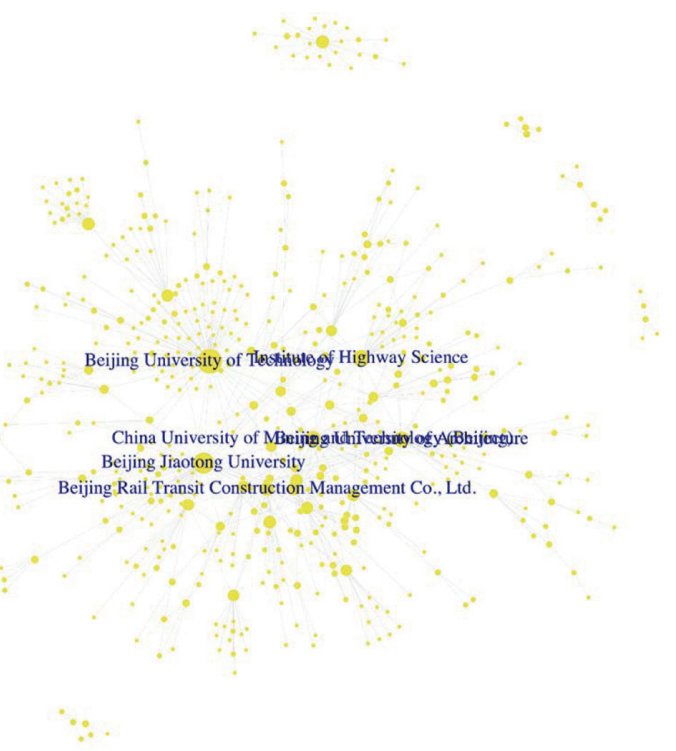

FIgURE 15: Community in the field of municipal transportation.

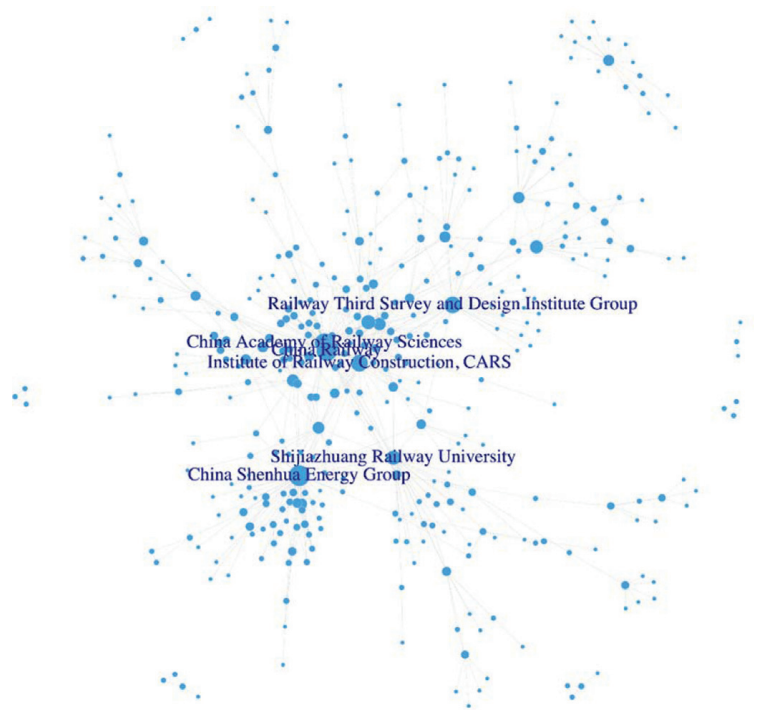

Figure 16: Community in the field of railway.

were all universities. Except these universities, important enterprise and research institute nodes included the Institute of Highway Science under the Ministry of Transport, Beijing Rail Transit Construction Management Co. Ltd., and Beijing Urban Construction Group Co. Ltd. These enterprises and research institutes were in the field of municipal transportation. From the geographical composition, $80 \%$ of organizations of community \#6 came from Beijing, 10\% from Tianjin, and $10 \%$ from Hebei Province, which reflected the significant geographical attribute of Beijing.

4.6. Railway Industry. Community \#9 (Figure 16) was in the field of the railway industry. Within the top ten nodes with the highest degree centrality, eight of them had relationships with railways and the other two nodes were in the field of energy. Since the railway industry in BTH Urban 
Agglomeration was mainly concerned with railway operations and electrical design, few enterprises in community \#9 were involved in manufacturing.

\section{Conclusions}

This article studies the coordinated development of Beijing-Tianjin-Hebei from the perspective of the community of innovation, which complements the previous research. Through investigation of the communities of innovation detected from the patent cooperation network in BTH Urban Agglomeration, we summarized four typical community structures and found significant industrial and geographical characteristics in the communities of innovation. Beijing, Tianjin, and Hebei have formed a patent cooperation network centered on universities and large state-owned enterprises mainly in six industry fields. Tianjin and Hebei both have close patent cooperation relationships with Beijing; however, there is a lack of cooperation between Tianjin and Hebei. Universities and state-owned enterprises played an important role in connecting organizations among regions; especially, universities have more potential to connect organizations from different regions due to their advantages in various research fields and nonprofit attributes. In the field of railway, electric power and petroleum BTH Urban Agglomeration had more connections in patent application compared with other industries. In the future, Beijing, Tianjin, and Hebei should further strengthen innovation cooperation and remove obstacles that hinder innovation.

\section{Data Availability}

The China Patent Database and Business Registration Database used to support the findings of this study were supplied by LIIN DATA under license and so cannot be made freely available. Requests for access to these data should be made to LIIN DATA via e-mail guochunlei@ liindata.com. The Tianyan check data can be obtained through the data query port: https://www.tianyancha. $\mathrm{com} /$.

\section{Disclosure}

Fang Zhou and Bo Zhang contributed to conceptualization, data curation, formal analysis, methodology, software, validation, visualization, and writing the original draft. Fang Zhou contributed to writing, review, and editing.

\section{Conflicts of Interest}

The authors declare that they have no conflicts of interest.

\section{Acknowledgments}

This work was supported by Beijing Municipal Education Commission (Grant no. SM201710038013).

\section{References}

[1] D. X. Yao, "Does the syphonage effect exist in the cooperative innovation of the BTH metropolitan region: from a comparative perspective with the pearl river delta," Economic Theory and Business Management, vol. 9, pp. 89-97, 2019.

[2] C. Liu, C. Niu, and J. Han, "Spatial dynamics of intercity technology transfer networks in China's three urban agglomerations: a patent transaction perspective," Sustainability (Switzerland), vol. 11, no. 6, 2019.

[3] M. Chen, Y. Qin, and N. Li, "Dynamic evolution analysis of cross-regional knowledge flow and innovation cooperation network," Studies in Science of Science, vol. 37, no. 12, pp. 2252-2264, 2019.

[4] W. Shi, W. Yang, and D. Du, "The scientific cooperation network of chinese scientists and its proximity mechanism," Sustainability (Switzerland), vol. 12, no. 2, 2020.

[5] L. Lyu, G. Meng, R. Huang, and F. Sun, "Spatial evolution and organization of urban agglomeration innovation network: a case study of beijing-tianjin-hebei urban agglomeration," Areal Research and Development, vol. 38, no. 1, pp. 50-55, 2019.

[6] H. Xing and C. Zhang, "Emerging of innovation network of urban agglomerations: the patent cooperation and the evolvement of the network of beijing-tianjin-hebei urban agglomerations," Areal Research and Development, vol. 37, no. 4, pp. 61-66, 2018.

[7] E. Coakes and P. Smith, "Developing communities of innovation by identifying innovation champions," The Learning Organization, vol. 14, no. 1, pp. 74-85, 2007.

[8] M. Lim and B. Y. Ong, "Communities of innovation," International Journal of Innovation Science, vol. 11, no. 3, pp. 402-418, 2019.

[9] Yi Su and T. Li, "Simulation analysis of knowledge transfer in a knowledge alliance based on a circular surface radiator model," Complexity, vol. 2020, Article ID 4301489, 27 pages, 2020.

[10] K. Fichter, "Innovation communities: the role of networks of promotors in open innovation," R\&D Management, vol. 39, no. 4, pp. 357-371, 2009.

[11] L. Fleming and D. M. Waguespack, "Brokerage, boundary spanning, and leadership in open innovation communities," Organization Science, vol. 18, no. 2, pp. 165-180, 2007.

[12] P. A. Gloor, R. Laubacher, S. Dynes, and Y. Zhao, "Visualization of communication patterns in cooperative innovation networks - analysis of some W3C working groups," in Proceedings of the twelfth international conference on Information and knowledge management (CIKM '03), pp. 56-60, Association for Computing Machinery, November 2003, New York, NY, USA.

[13] P. A. C. Smith, "Knowledge sharing and strategic capital," The Learning Organization, vol. 12, no. 6, pp. 563-574, 2005.

[14] Y. Kajikawa and Y. Takeda, "Structure of research on biomass and bio-fuels: a citation-based approach," Technological Forecasting and Social Change, vol. 75, no. 9, pp. 1349-1359, 2008.

[15] N. Shibata, Y. Kajikawa, Y. Takeda, and K. Matsushima, "Detecting emerging research fronts based on topological measures in citation networks of scientific publications," Technovation, vol. 28, no. 11, pp. 758-775, 2008.

[16] N. Shibata, Y. Kajikawa, Y. Takeda, I. Sakata, and K. Matsushima, "Detecting emerging research fronts in regenerative medicine by the citation network analysis of scientific publications," Technological Forecasting and Social Change, vol. 78, no. 2, pp. 274-282, 2011. 
[17] K. Nakai, H. Nonaka, A. Hentona et al., "Community detection and growth potential prediction using the stochastic block model and the long short-term memory from patent citation networks," in IEEE International Conference on Industrial Engineering and Engineering Management (IEEM), pp. 1884-1888, Bangkok, Thailand, December 2018.

[18] A. Clauset, M. E. J. Newman, and C. Moore, "Finding community structure in very large networks," Physical Review E, vol. 70, Article ID 066111, 2004.

[19] P. Pons and M. Latapy, "Computing communities in large networks using random walks," Journal of Graph Algorithms and Applications, vol. 10, no. 2, pp. 284-293, 2004.

[20] M. Rosvall and C. T. Bergstrom, "An information-theoretic framework for resolving community structure in complex networks," Proceedings of the National Academy of Sciences, vol. 104, no. 18, pp. 7327-7331, 2007.

[21] U. N. Raghavan, R. Albert, and S. Kumara, "Near linear time algorithm to detect community structures in large-scale networks," Physical Review E, vol. 76, no. 3, Article ID 036106, 2007.

[22] V. D. Blondel, J. L. Guillaume, R. Lambiotte, and E. Lefebvre, "Fast unfolding of communities in large networks," Journal of Statistical Mechanics: Theory and Experiment, vol. 2008, no. 10, Article ID p10008, 2008.

[23] J. Reichardt and S. Bornholdt, "Statistical mechanics of community detection," Phys Rev E, vol. 74, Article ID 016110, 2006.

[24] S. Tripathi, S. Moutari, M. Dehmer et al., "Comparison of module detection algorithms in protein networks and investigation of the biological meaning of predicted modules," Bmc Bioinformatics, vol. 17, no. 1, p. 129, 2016.

[25] Z. Yang, R. Algesheimer, and C. J. Tessone, "A comparative analysis of community detection algorithms on artificial networks," Scientific Reports, vol. 6, 2016.

[26] E. Eaton and R. Mansbach, "A spin-glass model for semisupervised community detection," in Proceedings of the Twenty-Sixth AAAI Conference on Artificial Intelligence AAAI'12, pp. 900-906, Toronto, Ontario, Canada, July 2012.

[27] L. Pan, C. Wang, and J. Xie, "A spin-glass model based local community detection method in social networks," in Proceedings of the IEEE 25th International Conference on Tools with Artificial Intelligence, pp. 108-115, Herndon, VA, USA, November 2013.

[28] R. S. Burt, Structural Holes: The Social Structure of Competition, Harvard University Press, Cambridge, MA, USA, 1st edition, 1995.

[29] G. Ahuja and R. Katila, "Technological acquisitions and the innovation performance of acquiring firms: a longitudinal study," Strategic Management Journal, vol. 22, no. 3, pp. 197-220, 2001.

[30] M. Makri, M. A. Hitt, and P. J. Lane, "Complementary technologies, knowledge relatedness, and invention outcomes in high technology mergers and acquisitions," Strategic Management Journal, vol. 31, no. 6, pp. 602-628, 2010.

[31] M. Meyborg, The role of German universities in a system of joint knowledge generation and innovation: a social network analysis of publications and patents with a focus on the spatial dimension, KIT Scientific Publishing, Karlsruhe, Germany, 2013.

[32] R. Huggins, D. Prokop, and P. Thompson, "Universities and open innovation: the determinants of network centrality," The Journal of Technology Transfer, vol. 45, no. 3, pp. 718-757, 2020 . 